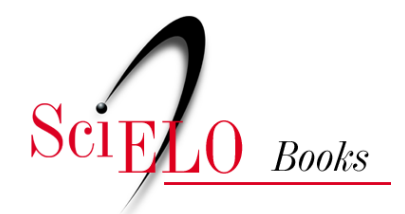

\title{
EdUfSCar
}

\section{5 - Modelagem computacional de líquidos iônicos}

\author{
Luciano T. Costa \\ Eudes Eterno Fileti
}

\section{SciELO Books / SciELO Livros / SciELO Libros}

COSTA, L.T., and FILETI, E.E. Modelagem computacional de líquidos iônicos. In: FREITAS, L.C.G., and OLIVEIRA, G.S., orgs. Aplicações de química teórica no estudo de materiais: métodos in silico para nanomateriais [online]. São Carlos: EdUFSCar, 2018, pp. 158-184. ISBN 978-65-8021612-3. Avalaible from: doi: 10.7476/9786580216123.0006. Also available in ePUB from: http://books.scielo.org/id/nvnjd/epub/freitas-9786580216123.epub.

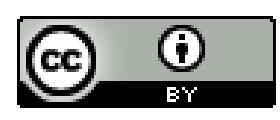

All the contents of this work, except where otherwise noted, is licensed under a Creative Commons Attribution $\underline{4.0 \text { International license. }}$

Todo o conteúdo deste trabalho, exceto quando houver ressalva, é publicado sob a licença Creative Commons Atribição 4.0. 


\section{MODELAGEM COMPUTACIONAL DE LÍQUIDOS IÔNICOS}
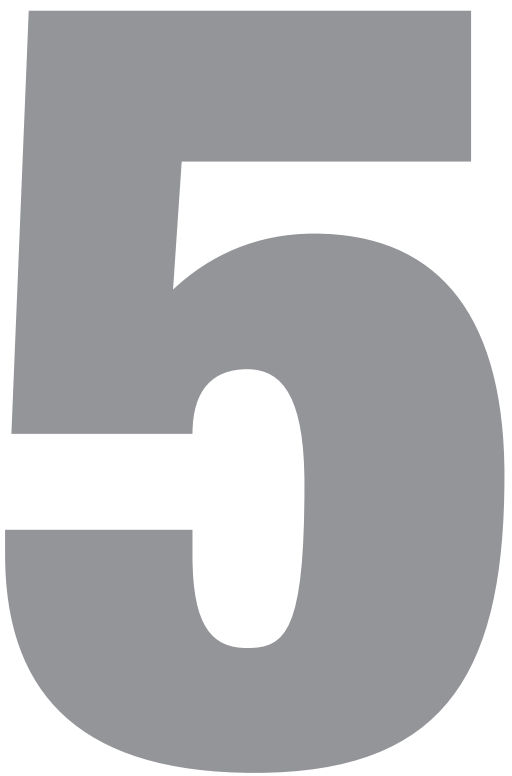

Luciano T. Costa $^{1}$ e Eudes Eterno Fileti ${ }^{2}$

1 Instituto de Química do Departamento de Físico-Química da Universidade Federal Fluminense (UFF), Niterói-RJ. E-mail: $<$ ltcosta@id.uff.br>.

2 Instituto de Ciência e Tecnologia da Universidade Federal de São Paulo (Unifesp), São José dos Campos-SP. E-mail: $<$ fileti@iq.usp>. 


\subsection{INTRODUÇã̃ $0^{3}$}

Líquidos iônicos (LIs) consistem em uma classe de solventes com propriedades ajustáveis e têm sido amplamente utilizados como substituto "verde" para solventes orgânicos tóxicos, perigosos, inflamáveis e altamente voláteis. ${ }^{4}$ De fato, as potenciais aplicações técnicas e comerciais de LIs estão principalmente ligadas às suas propriedades físico-químicas únicas, como baixa pressão de vapor, múltiplas interações de solvatação com compostos orgânicos e inorgânicos, ${ }^{5}$ excelente estabilidade química e térmica, ${ }^{6}$ relativa condutividade iônica e ampla janela eletroquímica. ${ }^{7}$ Além disso, propriedades como viscosidade, hidrofobicidade, densidade e solubilidade podem ser ajustadas, escolhendo diferentes combinações de cátions e ânions, para adequar o LI a exigências específicas. Tais características conferem aos LIs a capacidade de serem moldáveis, com potenciais aplicações científicas e tecnológicas. ${ }^{8}$

Ao longo da última década, os LIs apresentaram enorme potencial como (co) solventes e/ou reagentes em uma ampla gama de aplicações, incluindo a Engenharia Química (separação, extração e membranas), ${ }^{9}$ Química (síntese orgânica, reações catalíticas, síntese de nanomateriais e reações de polimerização), ${ }^{10}$ conversão de energia (baterias, células de combustível e armazenamento de calor), ${ }^{11}$ biotecnologia

Os autores agradecem ao suporte das agências de amparo à pesquisa CNPq e FAPESP. Brooks (2014), Mohammad e Inamuddin (2014), Weingärtner (2008), Reichardt (2005), Feng et al. (2010).

Weingärtner (2008), Reichardt (2005), Feng et al. (2010).

Weingärtner (2008), Reichardt (2005), Feng et al. (2010).

Brooks (2014), Mohammad e Inamuddin (2014), Weingärtner (2008), Reichardt (2005), Feng et al. (2010).

Hywel et al. (2014), Vishal Govind et al. (2013), McCrary et al. (2013), Azevedo et al. (2013).

Rao et al. (2013).

0 Brooks (2014), Mohammad e Inamuddin (2014).

11 Brooks (2014), Mohammad e Inamuddin (2014). 
(biocatálise, purificação de biomoléculas e produção de biocombustíveis) ${ }^{12}$ e ciências farmacêuticas (pró-drogas, transporte de drogas e solubilização). ${ }^{13}$

Do ponto de vista molecular e estrutural, os líquidos iônicos são compostos de cátions orgânicos de baixa simetria e ânions orgânicos ou inorgânicos de alta simetria. A primeira geração de líquidos iônicos, proposta no início da década de 1970 e meados de 1980, apresenta uma gama de possíveis compostos constituídos, em geral, por um cátion orgânico e um ânion inorgânico, tendo aplicações industriais relevantes: dispositivos eletroquímicos, como capacitores, solventes em síntese orgânica, aditivos em lubrificantes, entre outros. Propriedades físicas, como viscosidade, condutividade e densidade, têm sido alvo de investigação para esses compostos. ${ }^{14}$ Uma segunda geração permitiu o modelamento de propriedades químicas e físicas por meio da substituição de um ânion inorgânico por uma classe de ânions orgânicos funcionais ou pela substituição do cátion, promovendo características diferenciadas quanto à solvatação, à hidrofobicidade e à indução quiral. ${ }^{15}$ Recentemente, uma terceira geração de líquidos iônicos tem sido proposta, em que propriedades de interesse biológico podem ser modeladas com a proposição de novos cátions e ânions biocompatíveis, bioativos e não tóxicos, sendo compostas de líquidos iônicos com funcionalidade específica. ${ }^{16} \mathrm{~A}$ evolução dessas gerações revela a capacidade de racionalizar e planejar novos líquidos iônicos com base no conhecimento estrutural, termodinâmico e dinâmico desses compostos. ${ }^{17}$

Como exemplo, a forma e o tamanho do cátion, bem como sua assimetria, são responsáveis pela redução da temperatura de fusão dos líquidos iônicos, que é, por definição, abaixo de $100^{\circ} \mathrm{C}$. Além disso, cátions de baixa simetria possuem um empacotamento íon-íon menos eficiente, diminuindo a energia de rede e, portanto, o ponto de fusão. Um exemplo típico de cátions com essa característica são os do tipo alquilimidazólio. O tamanho e a forma dos cátions também são relevantes na determinação do ponto de fusão de líquidos iônicos; em geral, íons grandes provocam redução no ponto de fusão. Por outro lado, ânions são estruturas orgânicas ou inorgânicas menores que os cátions e geralmente são responsáveis pelas propriedades físicas do líquido. Esses ânions possuem carga negativa difusa, como o $\left[\mathrm{BF}_{4}\right]^{-} \mathrm{e}$ $\mathrm{o}\left[\mathrm{PF}_{6}\right]^{-}$, por exemplo. Com o aumento do tamanho do ânion, em geral, observa-se uma redução no ponto de fusão. Na Figura 5.1 apresenta-se a estrutura de alguns pares iônicos que constituem os líquidos iônicos mais convencionais.

Por outro lado, constata-se o uso da simulação computacional no auxílio a técnicas experimentais, a técnica preditiva, capaz de prever comportamentos, bem como propriedades físico-químicas. De fato, estudos revelam que a funcionalização de líquidos iônicos promove uma modulação de suas propriedades, derivadas dos

2 Rao et al. (2013), Huiyong et al. (2013).

13 Cojocaru et al. (2013), Suruchi et al. (2012), Stoimenovski et al. (2012), Bica et al. (2012).

14 Pádua et al. (2007), Greaves et al. (2006).

15 Welton (1999), Huddleston et al. (1998).

16 Hough et al. (2007).

17 Id. ibid. 
grupos funcionais substituintes, como éter, éster, álcool, amina etc. Como exemplo, recente estudo mostra que o grupo éter inserido na cadeia do cátion imidazólio diminui a viscosidade do líquido e aumenta a condutividade iônica dos eletrólitos. ${ }^{18}$

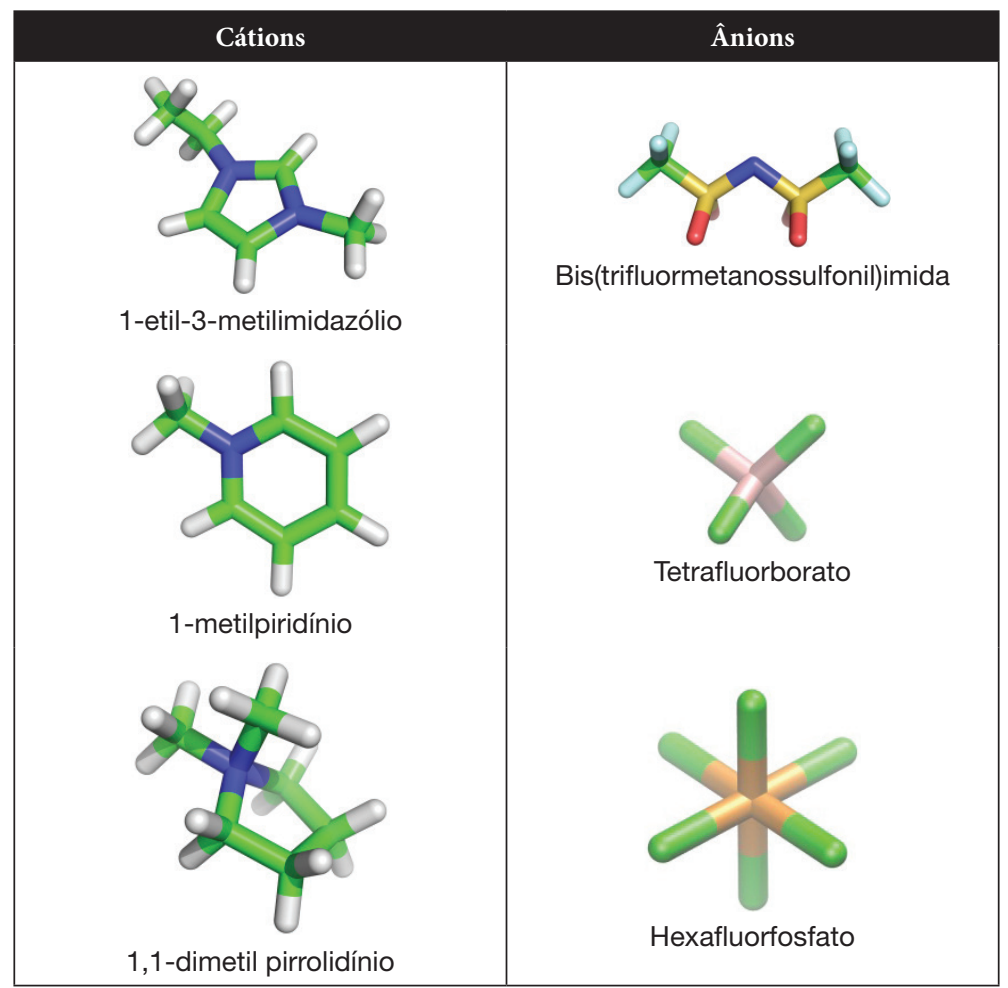

Figura 5.1 Modelos moleculares para alguns cátions e ânions mais empregados em simulação computacional de líquidos iônicos.

\subsection{Metodologias computacionais}

Nesta seção apresentar-se-á uma visão geral sobre duas das mais importantes abordagens computacionais para o estudo da matéria desordenada, em especial dos líquidos iônicos: a dinâmica molecular atomística e a dinâmica molecular coarse-grained. 


\subsection{DinÂMICA MOLECULAR ATOMÍSTICA}

Simulações computacionais têm sido, nas últimas décadas, ferramenta poderosa para tal estudo, especialmente quando se consideram escalas de tempo e de tamanho dos múltiplos processos associados à química supramolecular. ${ }^{19} \mathrm{~A}$ produção de configurações moleculares via dinâmica molecular é obtida por meio da solução das equações de Newton para cada uma das moléculas do sistema, a partir das posições e velocidades iniciais e do conhecimento da força que atua em cada molécula, em um determinado instante. ${ }^{20}$ Calculam-se, assim, as posições e velocidades de cada molécula ao fim de intervalos de tempo sucessivos, ou seja, obtém-se a evolução temporal do sistema. As forças que atuam na molécula i podem ser obtidas por meio de: ${ }^{21}$

$$
\mathrm{F}=-\frac{\mathrm{dU}(\mathrm{r})}{\mathrm{dr}}=-\mathrm{m}_{\mathrm{i}} \frac{\mathrm{d}^{2} \mathrm{r}_{\mathrm{i}}}{\mathrm{dt}^{2}}
$$

em que $U(\boldsymbol{r})$ é a energia potencial total das interações entre as moléculas de massa mi e que depende apenas do módulo $r$ da distância entre elas. Frequentemente, basta escolher uma forma simples de $U(\boldsymbol{r})$.

A energia potencial é considerada o fator mais importante da simulação, pois a modelagem das interações entre as moléculas depende necessariamente da escolha de um modelo adequado para a descrição da energia potencial. A forma analítica da energia potencial consiste em um conjunto de parâmetros que são característicos do sistema atômico-molecular que está sendo simulado e que são denominados campos de força empíricos. ${ }^{22}$

Campos de força descrevem a energia total de um sistema em função das contribuições das interações intramoleculares (interações ligadas) e intermoleculares (interações não ligadas). As interações intramoleculares compreendem, basicamente, o estiramento de ligações químicas, as deformações angulares e os termos torcionais, enquanto as interações intermoleculares são interações de Van der Waals e coulombianas. ${ }^{23}$ No caso das interações ligadas, são utilizadas aproximações de potencial de interação harmônico. Para a escolha do método clássico, é possível

19 Srivastava e Voth (2013), Voth (2012), Barone (2011), Schmid (2010), Sansom et al. (2010), Shim e Kim (2009), Senn e Thiel (2009), Hwang et al. (2006, 2009), Ekkabut et al. (2008), Wang et al. (2007), Marrink et al. (2007), Lyynden-Bell et al. (2007), Ariga e Kunitake (2006).

20 Allen e Tildesley (1987), Leach (2001), Rapaport (1995).

21 Allen e Tildesley (1987), Leach (2001), Rapaport (1995).

22 Allen e Tildesley (1987), Leach (2001), Rapaport (1995).

23 Allen e Tildesley (1987), Leach (2001), Rapaport (1995). 
considerar uma série de campos de força disponíveis. Entre os exemplos mais populares estão os campos AMBER, ${ }^{24} \mathrm{CHARMM}^{25} \mathrm{GROMOS}^{26}$ e OPLS-AA. ${ }^{27}$

Tabela 5.1 Formas analíticas do campo de força para descrição das interações ligadas e não ligadas.

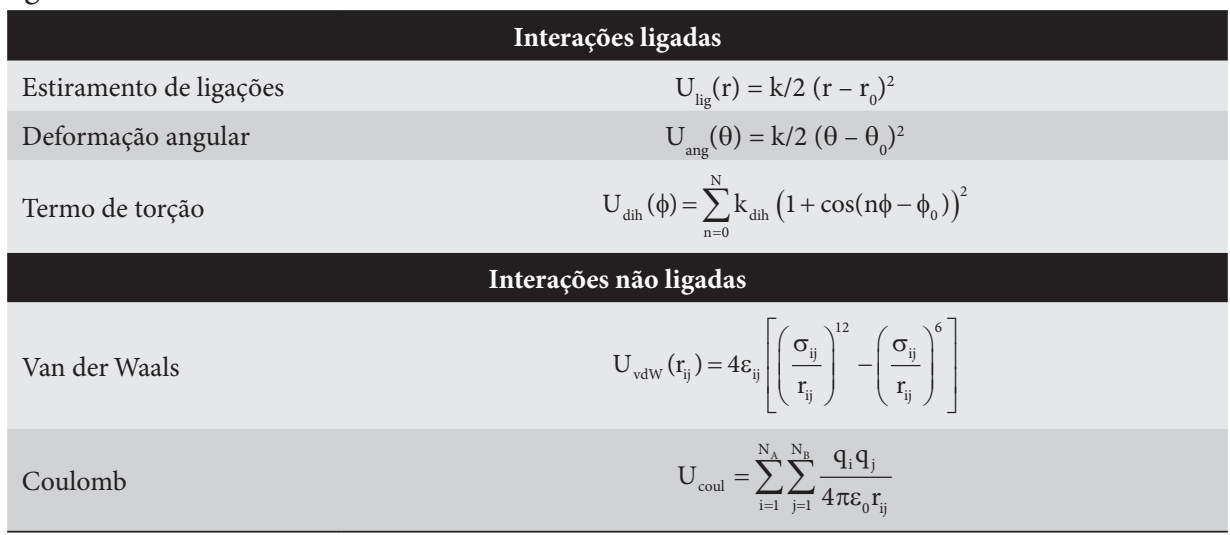

\subsection{CÁLCULO DE PROPRIEDADES}

Em geral, com exceção das fontes originais de livros altamente especializados, não é comum o leitor se deparar com o estado da arte sobre o cálculo de propriedades em dinâmica molecular. A proposta aqui é apresentar ao leitor uma descrição sumarizada da teoria que está por trás dos cálculos, cujos detalhes poderão ser acompanhados na literatura da área. ${ }^{28}$

\subsubsection{Estruturais}

A estrutura de líquidos pode ser analisada pela função de distribuição de pares de partículas i-j (átomos, moléculas ou íons) do sistema, $g(r):^{29}$

$$
g(r)=\rho^{-2}\left\langle\sum_{i} \sum_{i \neq j} \delta\left(r_{i}\right) \delta\left(r_{j}-r\right)\right\rangle=\frac{V}{N^{2}}\left\langle\sum_{i} \sum_{i \neq j} \delta\left(r-r_{i j}\right)\right\rangle
$$

\footnotetext{
Case et al. (2008).

MacKerell et al. (1998).

Soares et al. (2005).

Jorgensen et al. (1996).

Soares et al. (2005), Jorgensen et al. (1996), March e Tosi (1976).

Allen e Tildesley (1987), Sadus (1999), Hansen e McDonald (1990).
} 
em que $\mathrm{V}$ é o volume da caixa de simulação e $\mathrm{N}$ e $\mathbf{r}$ são o número e a posição das partículas, respectivamente. $O$ símbolo $\delta$ define a função delta de Dirac. ${ }^{30} \mathrm{~A}$ função $\mathrm{g}(\mathbf{r})$ contabiliza a densidade local de ocorrência de vizinhos $\mathrm{j}$ em torno de uma partícula i, normalizada pela densidade bulk do sistema. A Figura 5.2(A) ilustra a estrutura de um líquido monoatômico. Pode-se representar essa probabilidade por meio de um gráfico de ocorrências em que a partícula i está localizada na origem (em vermelho, na Figura 5.2), em que o eixo x define a distância $r$ entre uma dada partícula j de sua vizinha i, como mostra a figura. É importante lembrar que a função de distribuição radial de pares pode ser obtida de experimentos de difração de raio-X e espalhamento de nêutrons. ${ }^{31}$
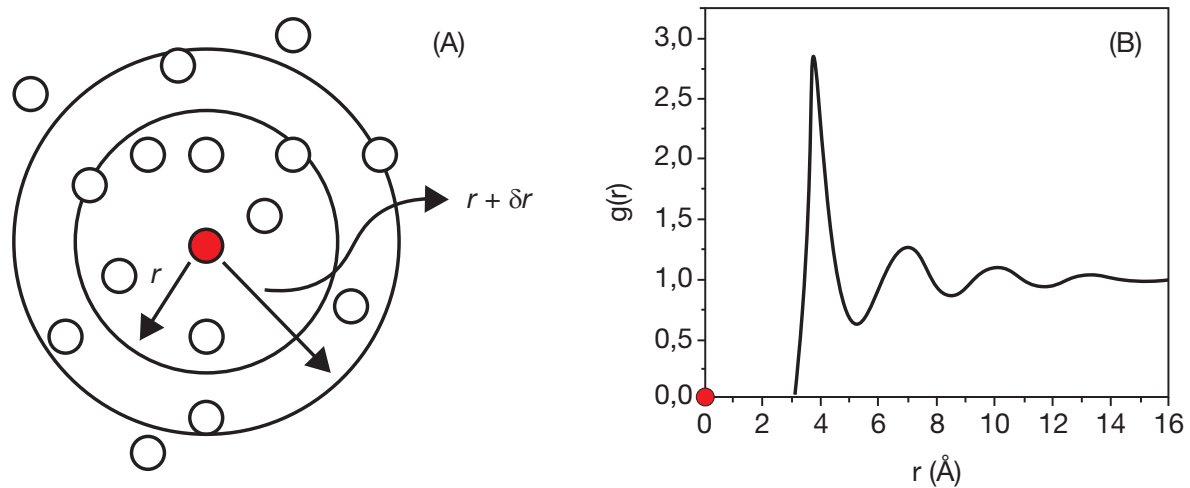

Figura 5.2 Representação de um líquido atômico, em que as esferas ilustram as partículas ou átomos do sistema e os círculos, as camadas (A). A distância $r$ define o raio da primeira camada de vizinhos, enquanto $\delta$ r é definido pela distância entre duas camadas consecutivas. A esfera no centro representa uma partícula i, e todas as outras, a vizinhança j. Ao lado, a g(r) correspondente (B).

Na Figura 5.2(B), o primeiro pico revela a distância de primeiros vizinhos j em torno da partícula i, como ilustrado na Figura 5.2(A). Outra ferramenta usada na caracterização estrutural e que ajuda a construir uma imagem da vizinhança local em torno de determinada espécie é o mapa de densidade de probabilidade. ${ }^{32}$ Essa análise é definida pela probabilidade de ocorrência de determinada espécie em torno de uma molécula de referência. Faz-se uma seleção de vizinhos em torno da molécula de referência, para a distância de primeiros vizinhos na $\mathrm{g}(\mathbf{r})$, salvando suas coordenadas $(\mathrm{x}, \mathrm{y}, \mathrm{z})$ e, dessa forma, construindo um mapa de densidade ao redor de uma dada partícula $\mathrm{i}$.

30 Hansen e McDonald (1990).

31 Hansen e McDonald (1990), Elliott (1952), March e Tosi (1976).

32 Urahata e Ribeiro (2004). 
A função $g(\mathbf{r})$ expressa a correlação de densidade local de equilíbrio. A densidade local em um ponto $r$ no espaço é expressa por: ${ }^{33}$

$$
\rho(r)=\sum_{i=1}^{N} \delta\left(r-r_{i j}\right)
$$

em que $\mathbf{r}$ é o vetor posição da partícula. Experimentalmente, obtém-se o fator de estrutura estático, $S(k)$, por espectroscopia de espalhamento de nêutrons e, a partir de sua transformada de Fourier, calcula-se a $g(\mathbf{r})$. Portanto, a densidade em termos do vetor de onda $\mathbf{k}$ é a transformada de Fourier da equação anterior: ${ }^{34}$

$$
\rho_{k}=\sum_{i=1}^{N} e^{-i k \cdot r_{i}}
$$

e a correlação de densidades no espaço recíproco, o fator de estrutura estático, a transformada de Fourier espacial da $g(\mathbf{r}):^{35}$

$$
\mathrm{S}(\mathrm{k})=\frac{1}{\mathrm{~N}}\left\langle\rho_{\mathrm{k}} \rho_{-\mathrm{k}}\right\rangle=\frac{1}{\mathrm{~N}}\left\langle\sum_{\mathrm{i}} \sum_{\mathrm{j}} \mathrm{e}^{-\mathrm{ik} \cdot\left(\mathrm{r}_{\mathrm{i}}-\mathrm{r}_{\mathrm{j}}\right)}\right\rangle
$$

em que $\boldsymbol{k}$ define o vetor de onda no espaço recíproco. O fator de estrutura estático é importante por facilitar a observação de correlação entre partículas em uma escala espacial mais estendida ou intermediária (intermediate range order, IRO), sendo expresso por um pré-pico em vetor de onda pequeno. A função $S(\boldsymbol{k})$ parcial pode ser definida pela equação: ${ }^{36}$

$$
\mathrm{S}(\mathrm{k})=\frac{1}{\sqrt{\mathrm{N}_{\alpha} \mathrm{N}_{\beta}}}\left\langle\sum_{\mathrm{i} \in \alpha}^{\mathrm{N}_{\alpha}} \sum_{\mathrm{i} \in \beta}^{\mathrm{N}_{\alpha}} \mathrm{e}^{-\mathrm{ik} \cdot\left(\mathrm{r}_{\mathrm{i}}-\mathrm{r}_{\mathrm{j}}\right)}\right\rangle
$$

em que $\alpha$ e $\beta$ representam espécies diferentes.

\subsubsection{Dinâmicas}

O avanço da mecânica estatística de não equilíbrio, na década de 1950, com os trabalhos realizados por Green e Kubo sobre fenômenos de transporte, deu início à formulação de funções de correlação na descrição de muitos processos dependentes do tempo. ${ }^{37}$ Assim, um microestado do sistema no espaço de fase pode ser definido

33 Hansen e McDonald (1990).

34 Id. ibid.

35 Id. ibid.

36 Id. ibid.

37 McQuarrie (2000). 
pela coordenada $\mathbf{r}$ e momentum p. Pode-se escrever, portanto, que a evolução no tempo se torna: ${ }^{38}$

$$
\begin{aligned}
& \mathrm{p}(\mathrm{t})=\mathrm{p}(\mathrm{p}, \mathrm{r} ; \mathrm{t}) \\
& \mathrm{r}(\mathrm{t})=\mathrm{r}(\mathrm{p}, \mathrm{r} ; \mathrm{t})
\end{aligned}
$$

Se for considerado que $\mathrm{A}\{\mathrm{p}(\mathrm{t}), \mathrm{r}(\mathrm{t})\}$ é função das coordenadas no espaço de fase, pode-se definir que: ${ }^{39}$

$$
\mathrm{A}\{\mathrm{p}(\mathrm{t}), \mathrm{r}(\mathrm{t})\}=\mathrm{A}\{\mathrm{p}, \mathrm{r} ; \mathrm{t}\}=\mathrm{A}(\mathrm{t})
$$

em que $A(t)$ define qualquer propriedade dependente do tempo para um dado sistema. Dessa forma, a função de correlação no tempo clássica de $A(t)$ pode ser definida por: ${ }^{40}$

$$
\mathrm{C}_{\mathrm{A}}(\mathrm{t})=\left\langle\sum_{\mathrm{i}=1}^{\mathrm{N}} \mathrm{A}_{\mathrm{i}}(\mathrm{t}) \mathrm{A}_{\mathrm{i}}(0)\right\rangle
$$

em que $\langle\ldots\rangle$ significa a média; $A_{\mathrm{i}}(t)$, o valor da propriedade $A$ em determinado tempo $t ; A_{\mathrm{i}}(0)$, o valor de $A$ em diferentes origens de tempo. Se $A(t)$ é uma função vetorial, a equação anterior se torna: ${ }^{41}$

$$
C_{A}(t)=\left\langle\sum_{i=1}^{N} A_{i}(t) \cdot A_{i}(0)\right\rangle
$$

Para sistemas como líquidos iônicos, está-se interessado em funções de correlação no tempo de propriedades, como velocidade das partículas i do sistema, orientação de vetores no espaço (função de correlação reorientacional), transições de ângulos diedros, entre outras. Por exemplo, a equação acima, em termos da velocidade de uma dada partícula $i$ do sistema, pode ser escrita como: ${ }^{42}$

$$
\mathrm{C}_{\mathrm{v}}(\mathrm{t})=\left\langle\mathrm{v}_{\mathrm{i}}(\mathrm{t}) \cdot \mathrm{v}_{\mathrm{i}}(0)\right\rangle
$$

em que, nesse caso, $C_{\mathrm{v}}(t)$ é conhecida como função de autocorrelação (ACF) da velocidade. A partir dessa função, é possível calcular o coeficiente de difusão, $D$, para uma dada partícula i, por meio da relação de Green-Kubo, em que: ${ }^{43}$

43 Hansen e McDonald (1990), McQuarrie (2000).
} 


$$
\mathrm{D}=\frac{1}{3} \int_{0}^{\infty}\left\langle\mathrm{v}_{\mathrm{i}}(\mathrm{t}) \cdot \mathrm{v}_{\mathrm{i}}(0)\right\rangle \mathrm{dt}
$$

O coeficiente de difusão pode ser obtido a partir da relação de Einstein, em que o deslocamento quadrático médio das partículas é definido por: ${ }^{44}$

$$
6 \mathrm{tD}=\left\langle\left|\mathrm{r}_{\mathrm{i}}(\mathrm{t})-\mathrm{r}_{\mathrm{i}}(0)\right|^{2}\right\rangle
$$

Essa função contém o autotermo do deslocamento de cada partícula $i$ do sistema em um dado tempo $t$. Os brackets denotam uma média no conjunto, que, em MD, significa uma média sobre todas as partículas $i$ e origens no tempo. Outra propriedade de transporte que pode ser calculada é a condutividade, também propriedade dos deslocamentos dos íons no sistema. Contudo, é uma propriedade coletiva de todos os íons, e, portanto, os termos cruzados devem ser contabilizados: ${ }^{45}$

$$
\kappa=\lim _{t \rightarrow \infty} \frac{e^{2}}{6 \mathrm{tVk}_{\mathrm{B}} \mathrm{T}}\left\langle\sum_{\mathrm{i}} \sum_{\mathrm{j}} \mathrm{z}_{\mathrm{i}} \mathrm{z}_{\mathrm{j}}\left[\mathrm{r}_{\mathrm{i}}(\mathrm{t})-\mathrm{r}_{\mathrm{i}}(0)\right]\left[\mathrm{r}_{\mathrm{j}}(\mathrm{t})-\mathrm{r}_{\mathrm{j}}(0)\right]\right\rangle
$$

em que $V$ é o volume; $k_{\mathrm{b}}$, a constante de Boltzmann; $T$, a temperatura; $e$, a carga do elétron; $z_{\mathrm{i}}$, a carga formal do íon $i$. Pode-se também, pela relação de Nernst-Einstein, estimar a condutividade do sistema por meio do coeficiente de difusão calculado pela equação acima:

$$
\mathrm{k}^{\mathrm{NE}}=\frac{\mathrm{e}^{2}}{\mathrm{k}_{\mathrm{B}} \mathrm{T}}\left(\rho_{-} \mathrm{z}_{-}^{2} \mathrm{D}_{-}+\rho_{+} \mathrm{z}_{+}^{2} \mathrm{D}_{+}\right)
$$

em que $\rho_{-}$e $p_{+}$são definidos como a densidade dos ânions e cátions na caixa de simulação; $z_{-}$e $z_{+}$, a carga formal dessas espécies; e $D_{-}$e $D_{+}$, os coeficientes de difusão de ânions e cátions, respectivamente. A razão $\kappa / \kappa_{\mathrm{NE}}$ é indicativa da formação de par iônico, revelando a importância da correlação de termos cruzados na dinâmica iônica.

Para ilustrar essas propriedades dinâmicas em sistemas contendo líquido iônico, têm sido aplicadas simulações MD em eletrólitos poliméricos derivados de líquidos iônicos com base no polímero polioxietileno (POE) ou poli(óxido de etileno), PEO, e ILs derivados do cátion imidazólio. ${ }^{46}$ De forma geral, simulações MD com potenciais não polarizáveis para ILs reportam propriedades dinâmicas, uma ordem de grandeza menor que dados experimentais. Por exemplo, a condutividade calculada do LI puro é de $3.2 \times 10^{-3}{\mathrm{~S} . \mathrm{cm}^{-1}}^{\text {, a }} 400 \mathrm{~K}$, enquanto o valor experimental é de $1.7 \times 10^{-2} \mathrm{~S} \mathrm{~cm}^{-1}$, a 373 K. Outras simulações MD reportam a mesma diferença entre condutividades calculada

44 McQuarrie (2000).

45 Hansen e McDonald (1990).

46 Siqueira e Ribeiro (2006), Costa e Ribeiro (2006). 
e experimental, ${ }^{47}$ por exemplo, em POE- $\mathrm{LiClO}_{4}$, cuja condutividade é uma ordem de grandeza menor em relação aos dados experimentais, comportamento similar ao encontrado em simulações MD para o sistema POE-LiI. Ainda assim, pode-se afirmar que esses resultados mostram modelos coerentes e transferíveis, já que foram utilizados em trabalhos anteriores realizados por Urahata e Siqueira. ${ }^{48}$

De forma a sintetizar a dinâmica de LIs em diferentes escalas de tempo, a Figura 5.3 mostra uma perspectiva em escala temporal das várias dinâmicas que ocorrem no sistema, evidenciando uma hierarquia dos processos dinâmicos do LI inserido na matriz polimérica. A rápida relaxação do grupo butil e oscilações na $\mathrm{C}_{\mathrm{v}}(\mathrm{t})$ ocorrem em escala de subpicossegundos, enquanto MSD e a $\mathrm{Cr}(\mathrm{t})$ ainda estão em um primeiro estágio de relaxação, dado por movimentos do tipo rattling e libracionais. O resultado mostrado na Figura 5.2 está em concordância com resultados anteriormente previstos na dinâmica de líquidos iônicos ${ }^{49} \mathrm{e}$ em correlação com a proposição de que, em transições de fase a partir da fase cristalina de LI, distintos graus de liberdade são ativados durante o processo de relaxação estrutural. Uma descrição em detalhe desses processos dinâmicos em líquidos iônicos derivados do cátion imidazólio pode ser vista na literatura. ${ }^{50}$
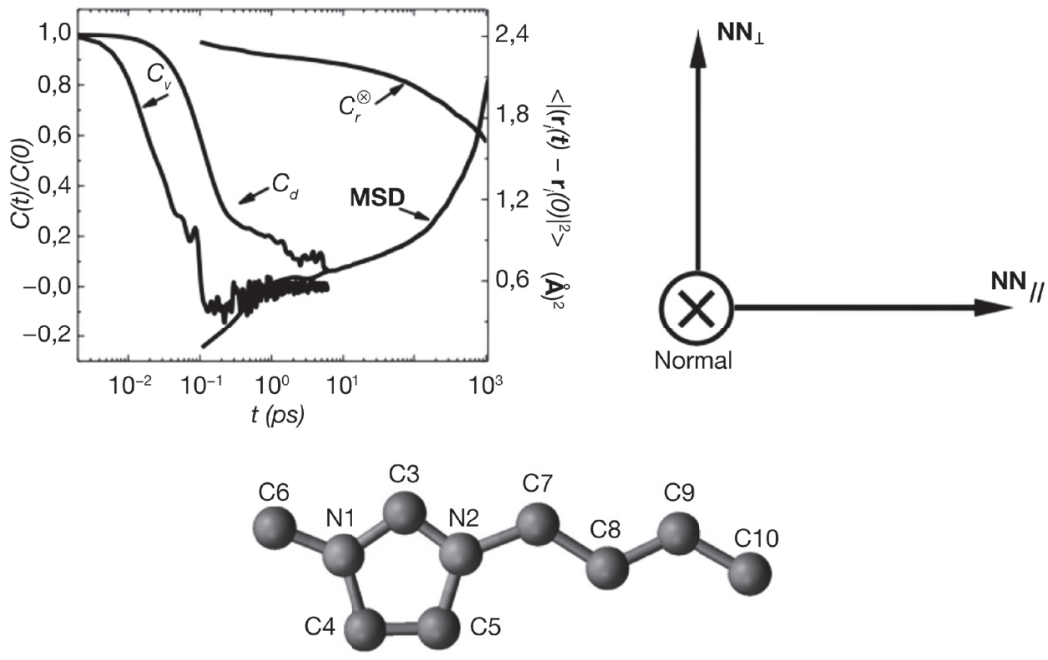

Figura 5.3 Comparação de algumas funções de correlação no tempo do cátion [bmim $]^{+}$no $\mathrm{P}(\mathrm{OE})_{8}$ - $[\mathrm{bmim}] \mathrm{PF}_{6}$. A figura mostra as ACFs de velocidade do centro de massa do cátion, $C_{\mathrm{v}}(t)$, do ângulo diedro da cadeia 1-butil, $C_{\mathrm{d}}(t)$, a projeção reorientacional normal ao plano do anel, $\mathrm{C}_{r}^{\otimes}$, e o MSD do centro de massa do cátion. Note que a escala do MSD está à direita, na figura.

\footnotetext{
Siqueira e Ribeiro (2006), Duan et al. (2005), Borodin e Smith (2006a, 2006b, 2006c).

Urahata e Ribeiro (2004, 2005), Siqueira e Ribeiro (2006).

Urahata e Ribeiro (2005, figura 11).

Id. $(2005,2006)$.
} 


\subsection{DINÂMICA MOLECULAR COARSE-GRAINED}

Apesar dos avanços computacionais, as simulações atomísticas ainda são limitadas a sistemas de tamanho reduzido em escala de tempo reduzida. Os processos celulares, no entanto, cobrem escalas de tempo de nanossegundos a segundos e envolvem centenas de diferentes moléculas que interagem em diferentes escalas de tamanho. Muitos fenômenos biologicamente interessantes, incluindo fusão de vesículas, formação de complexos de proteína de ordem mais elevada, enovelamento de proteínas e transdução de sinal, estão além da capacidade das simulações atomísticas. Para simular esses processos é necessária uma simplificação do modelo.

A utilização de modelos coarse-grained (CG) representa uma alternativa atraente comparada com modelos atomísticos, permitindo que simulações sejam executadas para sistemas maiores, em escalas de tempo mais longas, e ainda fornece detalhes estruturais realísticos. ${ }^{51}$ As funções analíticas para o campo de força atomístico e coarse-grained são exatamente as mesmas. O que difere nos dois campos são os parâmetros do potencial. Dinâmica molecular coarse-grained (DCG) é uma técnica de simulação relativamente recente e foi desenvolvida especificamente para modelar sistemas orgânicos de grande porte, na escala de tempo experimental. ${ }^{52} \mathrm{O}$ foco principal na DCG está nas interações que envolvem grandes deformações ou rearranjos de sistemas supramoleculares. Na DCG, agrupamentos de átomos são substituídos por um sítio interagente que tem a dimensão aproximada de quatro átomos pesados e interage com os sítios vizinhos ligados por interações tipo massa-mola, com constantes de força brandas que imitam o movimento da estrutura molecular. O uso dos sítios CG diminui os graus de liberdade do sistema, minimizando drasticamente, assim, o tempo computacional, em comparação com a dinâmica atomística. ${ }^{53}$ Além de diminuir o tempo por meio da redução do número de sítios de interação, conforme Figura 5.4, a DCG também permite aumentar o time step por uma ordem de grandeza, de forma que, no total, a DCG alcança duas ordens de grandeza a mais que a dinâmica atomística, permitindo, assim, que a DCG possa ser aplicada na investigação de problemas mais realísticos envolvendo materiais supramoleculares de amplo interesse biomédico e biotecnológico, como, por exemplo, problemas envolvendo membranas biomoleculares. ${ }^{54}$

Apesar da vasta aplicabilidade da dinâmica molecular, alguns fenômenos físico-químicos importantes não podem ser tratados diretamente por essa técnica. Entre esses fenômenos estão a quebra e a formação de ligações químicas, o que inviabiliza qualquer tentativa de estudo de reações químicas e efeitos quânticos de tunelamento.

51 Srivastava e Voth (2013), Voth (2012), Wang et al. (2007), Marrink et al. (2007), Barnoud et al. (2014).

52 Marrink et al. (2007).

53 Id. ibid.

54 Voth (2012), Hwang et al. (2009), Barnoud et al. (2014). 

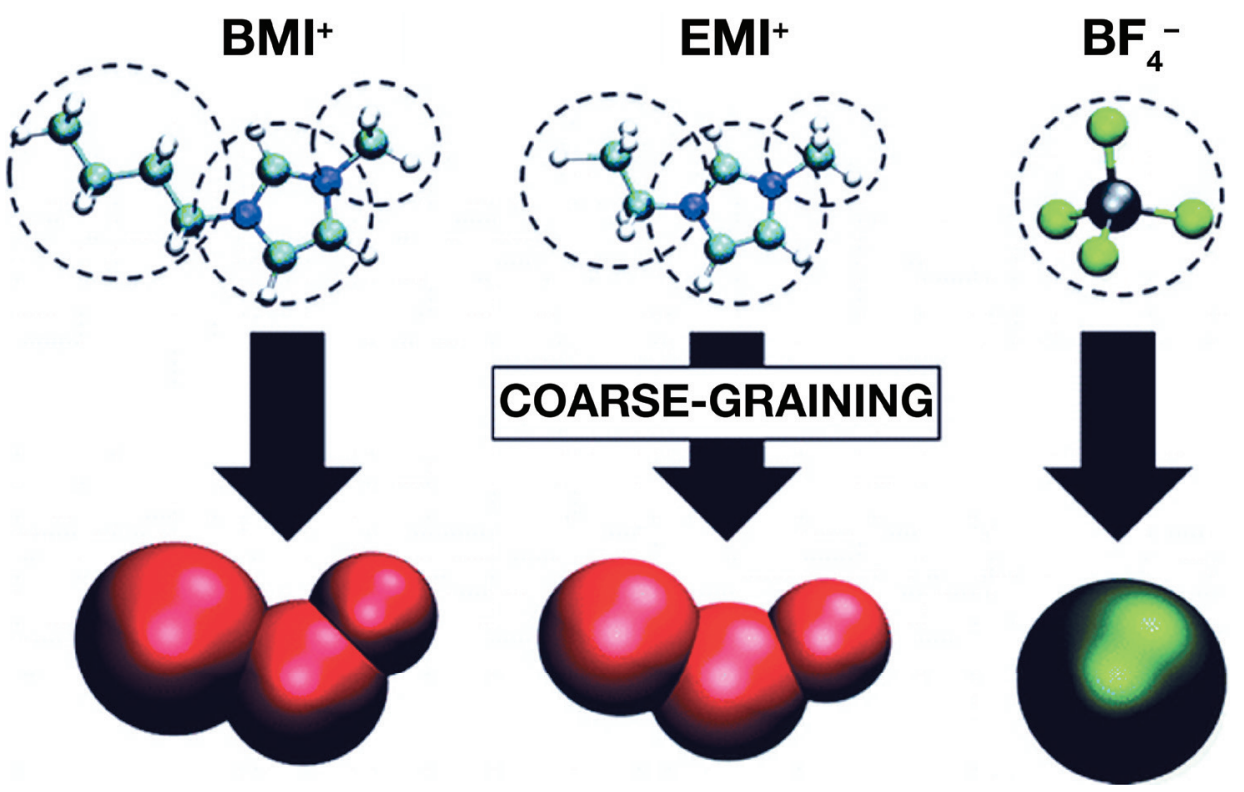

Figura 5.4 Representação da abordagem coarse-graining (embaixo) e atomística (em cima). Os 25 átomos do cátion $\mathrm{BMI}^{+}$são representados por três sítios no modelo $\mathrm{CG}$, o que reduz drasticamente o custo computacional da simulação. Reproduzida de Merlet et al. ${ }^{55}$

\subsection{MODELAGEM DE LÍQUIDOS IÔNICOS: APLICAÇÕES}

\subsubsection{Solvatação em líquidos iônicos}

Como mencionado, LIs podem dissolver muitos compostos orgânicos e inorgânicos que são insolúveis ou fracamente solúveis em água, bem como na maioria dos solventes orgânicos. De fato, na fase líquida, cátions e ânions formam uma complexa rede polar de ligações, em razão de suas intensas interações eletrostáticas, enquanto os grupos apolares formam domínios governados por interações de Van der Waals de curto alcance. ${ }^{56} \mathrm{~A}$ formação de domínios polares e apolares nos líquidos iônicos tem estabelecido uma nova maneira de analisar o processo de solvatação nesses ambientes. ${ }^{57}$ Nesse contexto, estudos computacionais recentes têm fornecido importantes análises das propriedades de solvatação em líquidos iônicos de nanoestruturas de carbono. Uma aplicação recente se refere ao problema da dissolução do fulereno $\mathrm{C}_{60} \mathrm{em}$ líquidos iônicos. Dissolver fulerenos de maneira eficiente é uma questão importante para numerosas aplicações. Uma enorme variedade de solventes (mais de 150) e de

55 Merlet et al. (2011).

56 Pádua et al. (2007), Lopes e Pádua (2006), Wang e Voth (2005).

57 Pádua et al. (2007), Wang e Voth (2005), Lopes e Pádua (2006), Lynden-Bell et al. (2007), Leach (2001). 
suas misturas tem sido aplicada para obtenção de soluções reais de fulerenos $\mathrm{C}_{60}, \mathrm{C}_{70} \mathrm{e}$ outros ainda maiores ${ }^{58}$ Os dados disponíveis na literatura ainda são, no entanto, pouco animadores. Na maioria dos solventes polares, como a água, soluções fulerênicas são extremamente diluídas ou são coloidais. Soluções relativamente concentradas são alcançadas apenas em alguns solventes de baixa polaridade, como os de hidrocarbonetos aromáticos e seus derivados halogenados. Por exemplo, a solubilidade do $\mathrm{C}_{60}$ em benzeno, tolueno e etilbenzeno é de $1,50 \mathrm{~g} \mathrm{~L}^{-1},{ }^{59} 2,40 \mathrm{~g} \mathrm{~L}^{-1},{ }^{60} 2,60 \mathrm{~g} \mathrm{~L}^{-1},{ }^{61}$ respectivamente, à temperatura de $298 \mathrm{~K}$. De forma geral, essas solubilidades ainda são muito baixas para a maioria das aplicações industriais relevantes e, dessa forma, líquidos iônicos surgem com uma possibilidade atrativa para contornar a alta hidrofobicidade do $\mathrm{C}_{60^{\circ}}$

A solvatação do $\mathrm{C}_{60}$ tem sido investigada computacionalmente, com o objetivo de se quantificar a complexa energética entre soluto e solvente, por meio da análise de sua energia potencial e energia livre. Além disso, o processo de dissolução do $\mathrm{C}_{60} \mathrm{em}$ líquidos iônicos tem sido analisado em termos da solubilidade. Simulações extensivas de dinâmica molecular para um aglomerado sólido de fulerenos $\mathrm{C}_{60}$, em bmimBF4, foram realizadas para tal investigação.
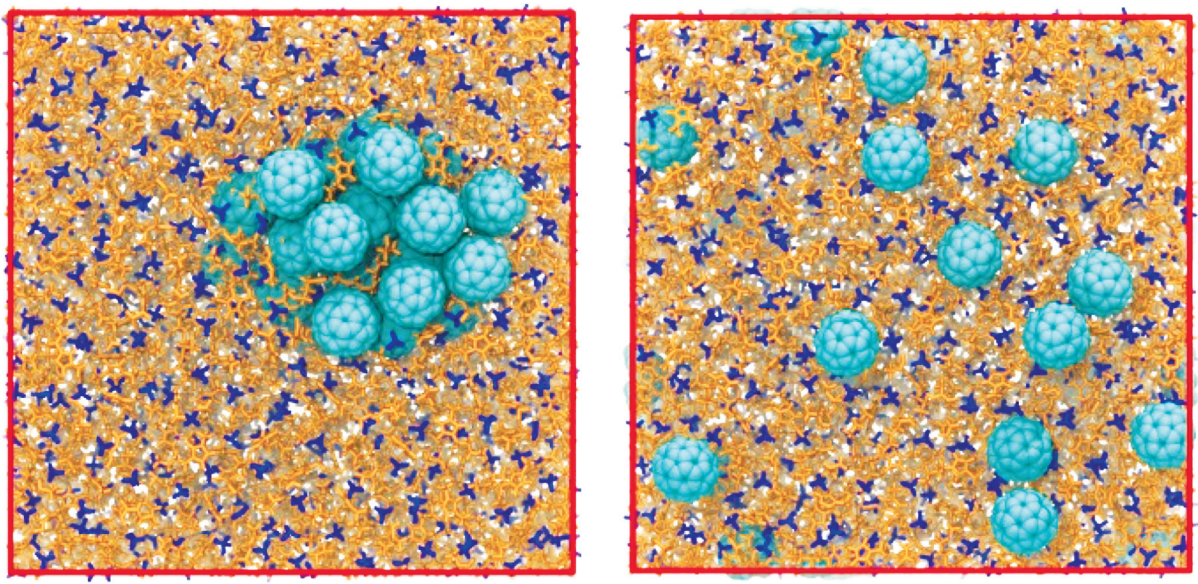

Figura 5.5 Imagens de configurações representativas do $\mathrm{C}_{60}$ em estado sólido (esquerda) e dissolvido (direita).

Embora a estrutura periódica bem ordenada do $\mathrm{C}_{60}$ em fase sólida se converta para um agregado em líquido iônico (semelhante ao da Figura 5.5), nenhuma molécula de fulereno é encontrada completamente dissolvida por um tempo significativo, durante $300 \mathrm{~ns}$ de evolução espontânea do sistema. Esse resultado está em excelente concordância com as recentes observações experimentais da solvatação do fulereno

58 Mchedlov-Petrossyan (2011), Semenov e Charykov (2010), Semenov et al. (2010).
59 Scrivens e Tour (1993).
60 Talukdar et al. (1997).
61 Scrivens e Tour (1993). 
$\mathrm{C}_{70}$ em líquidos iônicos comumente utilizados. ${ }^{62}$ Apesar do baixo poder de dissolução do líquido bmimBF4 em condições ambientes, esse solvente se torna excepcionalmente bem-sucedido, a partir de pequenos aumentos de temperatura da solução, sendo que cerca de $20 \mathrm{~K}$ já são suficientes para observar esse fenômeno. Para as temperaturas de 310,320 e $333 \mathrm{~K}$, as solubilidades obtidas são, respectivamente, 5, 49 e maiores que $66 \mathrm{~g} \mathrm{~L}^{-1}$. Uma vez que os fatores entálpicos não mudam significativamente entre 300 e $320 \mathrm{~K}$, a dissolução é impulsionada em razão do aumento do fator entrópico. Não é possível identificar com os modelos simulados a solubilidade máxima do $\mathrm{C}_{60}$ em temperaturas maiores, já que esses sistemas originalmente continham apenas 30 moléculas de fulereno, correspondendo a um máximo para a solubilidade de $66 \mathrm{~g} \mathrm{~L}^{-1}$. Esse valor-limite foi alcançado e excedido em $333 \mathrm{~K}$ e pode ser comparado à solubilidade em solventes "reativos", 63 embora nenhuma reação entre $\mathrm{o}_{60} \mathrm{e}$ o líquido iônico seja prevista.
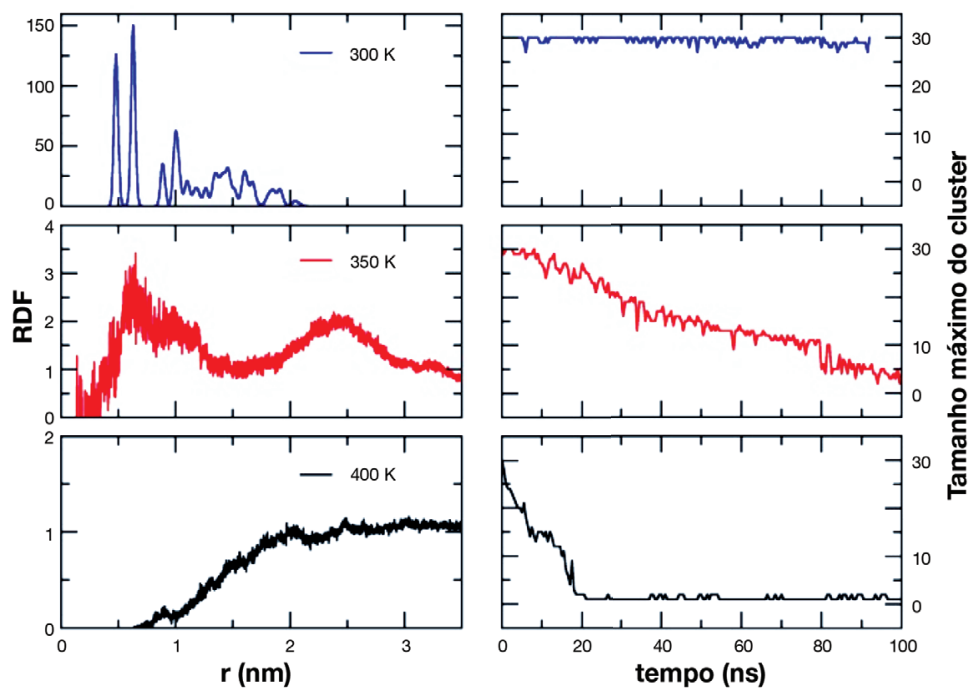

Figura 5.6 Função de distribuição radial em relação ao centro de massa do $\mathrm{C}_{60}$ (esquerda) e evolução do tamanho dos agregados em função do tempo de simulação (direita). Os picos pronunciados e bem ordenados da RDF em $300 \mathrm{~K}$, cujo arranjo é similarmente observado em baixas temperaturas (menores que $260 \mathrm{~K}$ ), deixam clara a existência de agregados moleculares não dissolvidos.

Esses resultados são bastante encorajadores e podem chamar atenção aos representantes de outras famílias de líquidos iônicos à temperatura ambiente, a fim de promover a solubilidade para valores cada vez maiores. A polarização mútua do bmimBF4 e do $\mathrm{C}_{60}$ desempenha um papel fundamental em dissoluções bem- 
-sucedidas. A natureza iônica do líquido bmimBF4 e a carga deslocalizada do cátion são importantes pré-requisitos para sua polarizabilidade eletrônica. Vale notar que essa aproximação é possível mesmo com líquidos iônicos à temperatura ambiente, mas impossível com soluções de íons em líquidos moleculares polares. Nesse último caso, íons são fortemente solvatados pelas moléculas de solvente e não conseguem se aproximar do fulereno. O fulereno é coordenado predominantemente por partículas neutras, as quais, na maioria dos casos, exibem de insignificante (álcoois, por exemplo) a negligenciável (nitrilas, por exemplo) potencial de polarização.

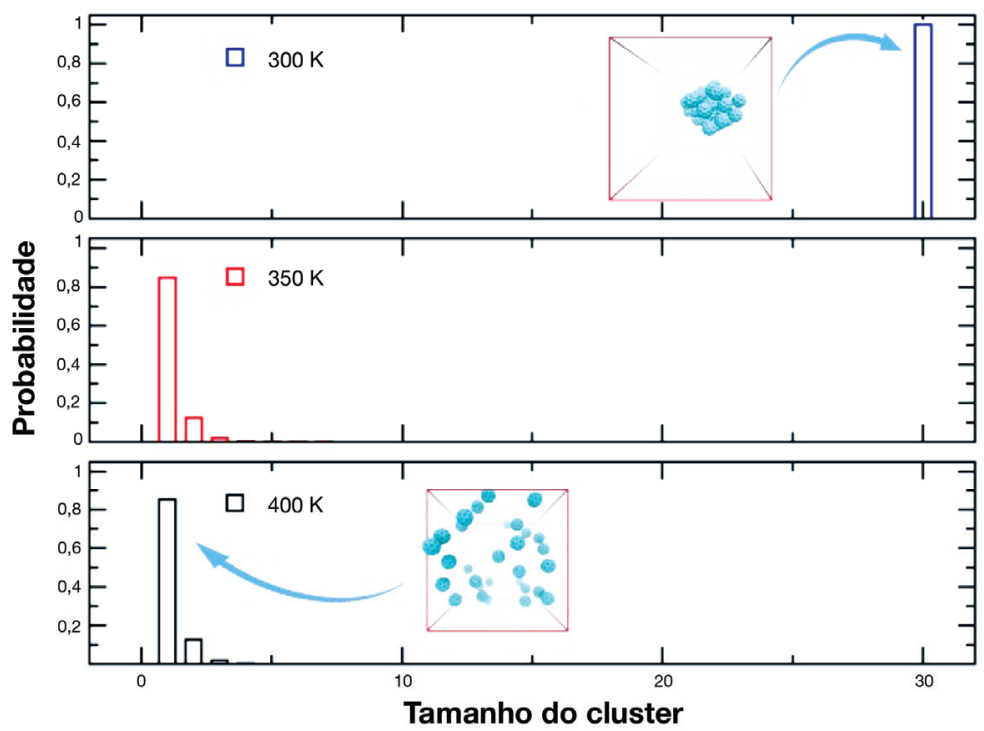

Figura 5.7 Monômeros, dímeros e trímeros do $\mathrm{C}_{60}$ em bmimBF4.

\subsubsection{Captura e separação de gases}

A concentração de dióxido de carbono na atmosfera continua a aumentar, já ultrapassando os níveis de 400 ppm, resultado da ação antropogênica, cujas principais fontes são a acelerada produção industrial, emissões por veículos automotivos e a queima de reservas florestais. Scott et al., ${ }^{64} \mathrm{em}$ recente trabalho de revisão, notaram a necessidade de uma política agressiva para a implementação de tecnologias de captura de carbono. De fato, como constatado por Zhang et al., novas tecnologias para captura de $\mathrm{CO}_{2}$ são necessárias, já que as atuais, com base em solventes orgânicos convencionais, como as soluções aquosas de metanolamina (MEA), possuem limitações e elevado custo energético ao fim do processo de captura ${ }^{65}$ Além

64 Scott et al. (2012).

65 Zhang, Lin e Wang (2008). 
disso, o $\mathrm{CO}_{2}$ não apenas contribui para a mudança climática como, não sendo ele utilizado na geração de compostos químicos por meio de sua conversão, abre, assim, um debate sustentável quanto ao seu real aproveitamento energético. Portanto, processos não apenas de captura, mas aliados com a conversão de $\mathrm{CO}_{2}$, tornam-se um atrativo do ponto de vista tecnológico.

Nesse ponto, torna-se importante salientar que os processos devem possuir tecnologia suficiente para não apenas capturar o $\mathrm{CO}_{2}$, mas o fazer de forma seletiva e específica, já que outros gases presentes são considerados interferentes no processo de captura, como $\mathrm{H}_{2} \mathrm{~S}$ e $\mathrm{SO}_{2}$, entre outros. As tecnologias emergentes de pós-combustão incluem sistemas com base em carbonato, amônia aquosa, membranas, adsorventes sólidos para captura, MOFs (Metal Organic Frameworks) e líquidos iônicos. ${ }^{66}$

Blanchard et al. publicaram um dos primeiros trabalhos sobre o assunto, no qual reportaram a alta solubilidade de $\mathrm{CO}_{2} \mathrm{em}$ [bmim][PF6], com fração molar de 0,6 em $8 \mathrm{MPa}$. A solubilidade de [bmim][PF6] em $\mathrm{CO}_{2}$ é da ordem de 10-5, a 13,8 $\mathrm{MPa}$ e $40{ }^{\circ} \mathrm{C}$. Após a aplicação de $8 \mathrm{MPa}$ de $\mathrm{CO}_{2}$, observou-se que a fase líquida aumenta em volume em apenas 10-20\%, comportamento esse diferente de solventes orgânicos, os quais possuem elevado aumento do volume quando é adicionado gás. A mudança de volume do líquido é medida pela seguinte relação:

$$
\frac{\Delta \mathrm{V}}{\mathrm{V}_{0}}(\%)=100 \frac{\mathrm{v}_{\mathrm{m}}(\mathrm{T}, \mathrm{p}, \mathrm{x})-\mathrm{V}_{\mathrm{IL}}\left(\mathrm{T}, \mathrm{p}_{0}\right)}{\mathrm{V}_{\mathrm{IL}}\left(\mathrm{T}, \mathrm{p}_{0}\right)}
$$

em que $\mathrm{V}_{\mathrm{m}}(\mathrm{T}, \mathrm{p}, \mathrm{x})$ é o volume molar na mistura de concentração $x$ de $\mathrm{CO}_{2}, \mathrm{eV}_{\mathrm{IL}}(\mathrm{T}$, $\mathrm{p}_{0}$ ) é o volume molar do líquido iônico puro. Shiflett e Yokozeki têm obtido resultados para a solubilidade de $\mathrm{CO}_{2}$ em $[\mathrm{bmim}]\left[\mathrm{PF}_{6}\right]$ e $[\mathrm{bmim}]\left[\mathrm{BF}_{4}\right]$ aplicando uma equação cúbica de estado em ajuste aos dados experimentais de absorção em diferentes temperaturas e pressões. ${ }^{67}$ Cálculos de dinâmica molecular de equilíbrio, usando o pacote GROMACS e condições termodinâmicas semelhantes ao trabalho de Shiflett e Yokozeki, variando a concentração de $\mathrm{CO}_{2}$ em líquido iônico [emim] [Tf2N], foram realizados. ${ }^{68} \mathrm{~A}$ Tabela 5.2 mostra um resumo das características dos sistemas simulados a $313 \mathrm{~K}$, e a Figura 5.8 revela que a variação de volume molar da mistura segue a mesma tendência encontrada pelos autores, validando não apenas o modelo computacional usado, mas predizendo o comportamento da solubilidade de $\mathrm{CO}_{2}$ nesse sistema.

66 Blanchard et al. (1999), Cadena et al. (2004), Li et al. (2011).

67 Shiflett e Yokozeki (2005).

68 Lourenço et al. (2013). 
Tabela 5.2 Dados de expansão do volume e variação do volume molar para o sistema $\operatorname{emim}[\mathrm{Tf} 2 \mathrm{~N}] / \mathrm{CO}_{2}$.

\begin{tabular}{cccccc}
$\begin{array}{c}\mathbf{x} \text { (fração } \\
\text { molar) } \mathbf{C O}_{\mathbf{2}}\end{array}$ & $\begin{array}{c}\mathbf{N} \\
\text { \#CO }\end{array}$ & $\begin{array}{c}\text { Volume molar } \\
\left(\mathbf{c m}^{3} / \mathbf{m o l}\right)\end{array}$ & $\mathbf{\Delta V / \mathbf { V } _ { \mathbf { 0 } }}$ & $\begin{array}{c}\text { Expansão do } \\
\text { volume }(\%)\end{array}$ & $\begin{array}{c}\text { Densidade } \\
\left(\mathbf{k g} / \mathbf{m}^{3}\right)\end{array}$ \\
\hline 0 & 0 & $127,3(0,06)$ & 0 & 0 & $1537(1)$ \\
0,03 & 10 & $124,5(0,11)$ & $-2,1$ & 0,9 & $1534(2)$ \\
0,059 & 20 & $122,1(0,09)$ & $-4,1$ & 1,9 & $1530(1)$ \\
0,099 & 35 & $118,4(0,03)$ & $-7,0$ & 3,2 & $1526(1)$ \\
0,135 & 50 & $115,2(0,07)$ & $-9,5$ & 4,6 & $1521(1)$ \\
0,206 & 83 & $108,9(0,03)$ & $-14,5$ & 7,7 & $1510(2)$ \\
0,286 & 128 & $101,8(0,03)$ & $-20,1$ & 11,9 & $1497(1)$ \\
0,351 & 173 & $95,9(0,01)$ & $-24,6$ & 16,1 & $1485(1)$ \\
0,389 & 204 & $92,4(0,02)$ & $-27,4$ & 18,9 & $1478(1)$ \\
\hline
\end{tabular}

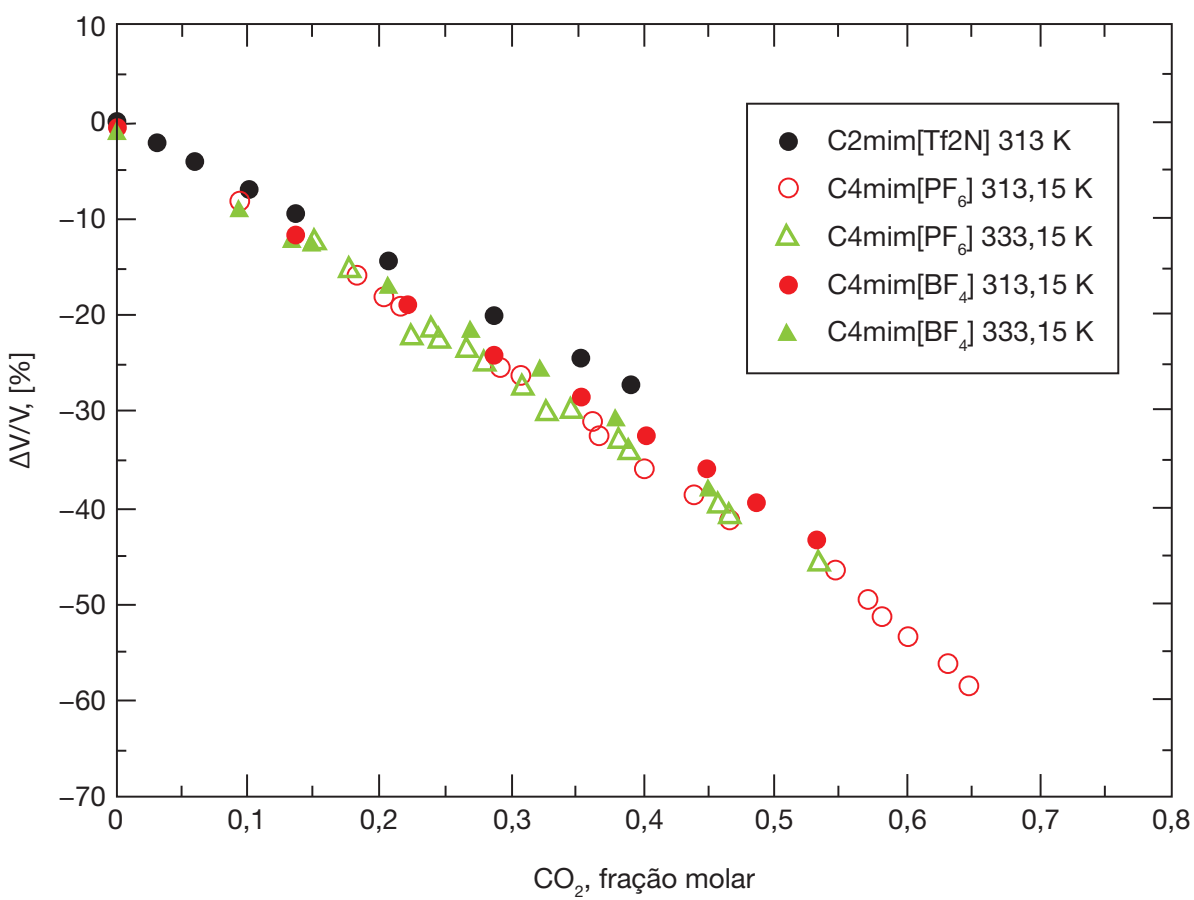

Figura 5.8 Expansão do volume molar $\left(\Delta \mathrm{V} / \mathrm{V} \_0\right)$ do sistema emim[Tf2N] (círculo preto) a $313 \mathrm{~K}$, a partir de cálculos de dinâmica molecular (MD), comparada com dados experimentais de Shiflett et al. para os sistemas bmim[BF4] e bmim[PF6]. ${ }^{69}$ 
$\mathrm{O}$ volume livre e o volume livre fracional (FFV, do inglês Fractional Free Volume) aumentam monotonicamente com a concentração de $\mathrm{CO}_{2}$. Lin e Freeman reportam que, para líquidos, os valores de volume livre fracional são tipicamente maiores que $21 \%$, enquanto para polímeros tendem a ser menores que esse valor. ${ }^{70}$ Como visualizado na Tabela 5.3, os valores de FFV se tornam mais próximos aos polímeros que aos líquidos regulares, podendo ser justificado pelas fortes interações de Coulomb e pela alta densidade. Mais detalhes acerca desse estudo podem ser encontrados na literatura. ${ }^{71}$

Tabela 5.3 Volume livre (FV, \%), volume fracional livre (FFV, \%) e desvios padrões entre parênteses.

\begin{tabular}{cccc|} 
Fração molar (x) & \#CO $_{2}$ & FV & FFV \\
\hline 0 & 0 & $37,4(0,3)$ & $18,6(0,2)$ \\
0,030 & 10 & $37,4(0,3)$ & $18,7(0,2)$ \\
0,059 & 20 & $37,6(0,3)$ & $18,9(0,2)$ \\
0,099 & 35 & $37,7(0,3)$ & $19,0(0,2)$ \\
0,135 & 50 & $37,8(0,3)$ & $19,1(0,1)$ \\
0,206 & 83 & $38,1(0,3)$ & $19,6(0,2)$ \\
0,286 & 128 & $38,5(0,3)$ & $20,0(0,2)$ \\
0,351 & 173 & $38,8(0,3)$ & $20,4(0,2)$ \\
0,389 & 204 & $39,0(0,3)$ & $20,6(0,2)$ \\
\hline
\end{tabular}

Medidas de solubilidade realizadas por Yim e Lim, em cinco líquidos iônicos, ${ }^{72}$ permitiram estabelecer a seguinte ordem: $[\mathrm{hmim}][\mathrm{Tf} 2 \mathrm{~N}]>[\mathrm{hmim}][$ triflato $]>$ $[\mathrm{hmim}]\left[\mathrm{PF}_{6}\right]>[\mathrm{hmim}]\left[\mathrm{BF}_{4}\right]>[\mathrm{hmim}]\left[\mathrm{MeSO}_{4}\right]$ a $333,15 \mathrm{~K}$. De acordo com os autores, a maior solubilidade associada ao $[\mathrm{hmim}][\mathrm{Tf} 2 \mathrm{~N}]$ se deve ao maior número de átomos de flúor, o que não ocorre com a espécie $\mathrm{MeSO}_{4}$. A molécula de $\mathrm{CO}_{2}$ apresenta momento de quadrupolo, interage com ânions por meio de interações do tipo ácido-base de Lewis e tem alta afinidade por flúor, com a formação espontânea de $\mathrm{F}^{-\mathrm{CO}_{2}}$.

Anthony et al. mediram a solubilidade de uma série de gases em $[\mathrm{bmim}]\left[\mathrm{BF}_{4}\right]$, $[\mathrm{bmim}][\mathrm{Tf} 2 \mathrm{~N}]$ e $[\mathrm{bmim}]\left[\mathrm{PF}_{6}\right] .{ }^{73} \mathrm{CO}_{2}$ e $\mathrm{NO}_{2}$ apresentaram as maiores solubilidades e interações mais fortes com os líquidos iônicos. A $25^{\circ} \mathrm{C}$, a constante de Henry para $\mathrm{CO}_{2}$ em $[\mathrm{bmim}]\left[\mathrm{PF}_{6}\right]$ é de $53,4 \pm 0,3$ bar, $[\mathrm{bmim}]\left[\mathrm{BF}_{4}\right]$ de $59,0 \pm 2,6$ bar e [bmim] [Tf2N] de 33,0 $\pm 0,3$ bar, com $\Delta \mathrm{H}$ de $(-14,3 ;-13,96 ;-12,5) \mathrm{kJ} / \mathrm{mol}$, respectivamente.

Lee et al. investigaram a influência de ânions halogenetos sobre a solubilidade de $\mathrm{SO}_{2}{ }^{74} \mathrm{~A}$ ordem determinada é $[\mathrm{emim}] \mathrm{Br}>[\mathrm{emim}] \mathrm{Cl}>[\mathrm{emim}] \mathrm{I}$, com razão mo$\operatorname{lar}\left(\mathrm{mol} \mathrm{SO}_{2} / \mathrm{mol} \mathrm{LI}\right)$ de 2,06, 2,11 e 1,91, respectivamente. A solubilidade determi-

\footnotetext{
70 Lin e Freeman (2005).

71 Lourenço et al. (2013).

72 Id. ibid.

73 Anthony et al. (2005).

74 Lee et al. (2010).
} 
nada para [emim] Cl (2,03 $\mathrm{mol} / \mathrm{mol}), \mathrm{hmimCl}(2,19 \mathrm{~mol} / \mathrm{mol})$ e omimCl $(2,19 \mathrm{~mol} /$ mol) indica o papel secundário do cátion sobre a solubilidade, enquanto o ânion exerce um papel primordial.

Estudos de Shiflett e Yokozeki mostraram, no entanto, que o $[\mathrm{bmim}]\left[\mathrm{PF}_{6}\right]$ não é necessariamente a melhor escolha para separação gasosa ou captura de $\mathrm{H}_{2} \mathrm{~S}$ e $\mathrm{CO}_{2}$, já que o LI pode afetar a seletividade do gás. ${ }^{75}$

Uma descrição em nível molecular das interações de LI com $\mathrm{SO}_{2}, \mathrm{CO}_{2}$ e $\mathrm{N}_{2}$ foi apresentada por Prasad e Senapati e suas implicações à solubilidade de gases. ${ }^{76}$ Resultados indicaram a importância da interação ânion-gás na solubilidade de gases em LI. A ordem de solubilidade encontrada é de $\mathrm{SO}_{2}>\mathrm{CO}_{2}>\mathrm{N}_{2}$. A interação ânion-gás segue a mesma ordem, sendo mais fortes as interações ânion- $\mathrm{SO}_{2}$, com cálculos realizados em nível de teoria MP2 e função de base 6-311+G*. Como exemplo, $\mathrm{PF}_{6}-\mathrm{SO}_{2}$ tem energia de interação de $-32,78 \mathrm{~kJ} / \mathrm{mol}, \mathrm{PF}_{6}-\mathrm{CO}_{2}$ é de $-11,08 \mathrm{~kJ} / \mathrm{mol}$ e $\mathrm{PF}_{6}-\mathrm{N}_{2}$ é de $-4,67 \mathrm{~kJ} / \mathrm{mol}$. Outros ânions estudados foram F-, Cl-, Br- e NO3-.

Em 2013, Haghbakhsh et al. propuseram uma correlação com base no método de regressão múltipla para predizer a solubilidade de $\mathrm{CO}_{2}$ em 27 líquidos iônicos comuns, a partir de 3.073 dados da literatura sobre o tema. ${ }^{77}$ Como exemplo, para o ânion triflato, foi obtida a seguinte ordem de solubilidade: [omim][TFA] $>$ [hmim] $[$ TFA $]>[$ bmim $][$ TFA $]>[$ emim $][$ TFA $]$. Ainda foram estudados líquidos iônicos com base em ânions $\mathrm{PF}_{6}^{-}, \mathrm{BF}_{4}^{-}$e cátions com base em piridínio, fosfônio, entre outros.

A densidade molar, que segue a ordem $[\mathrm{emim}][\mathrm{Tf} 2 \mathrm{~N}]>[$ bmim $][\mathrm{Tf} 2 \mathrm{~N}]>$ $[\mathrm{hmim}][\mathrm{Tf} 2 \mathrm{~N}]>[\mathrm{omim}][\mathrm{Tf} 2 \mathrm{~N}]$, pode ser associada à solubilidade, cujo comportamento é inverso. A explicação está no enfraquecimento da interação cátion-ânion de emim para omim, gerando um aumento de volumes livres no LI, com o consequente aumento do espaço livre disponível aos gases. ${ }^{78}$ De acordo com Cadena et al. ${ }^{79}$ a densidade menor de $[\mathrm{bmmim}]\left[\mathrm{PF}_{6}\right]$ em relação a $[\mathrm{bmim}]\left[\mathrm{PF}_{6}\right]$ sugere que o grupo metila reduz a eficiência de empacotamento. Resultados de dinâmica molecular indicam que o $\mathrm{CO}_{2}$ tem efeito desprezível para a organização de $\mathrm{PF}_{6}$ sobre o carbono $\mathrm{C} 2$, o que se deve às interações coulômbicas fortes responsáveis pela organização do líquido, forçando o gás a ocupar os interstícios, causando aumento da densidade molar do líquido iônico, já que não há expansão de volume.

De forma a evidenciar a localização do $\mathrm{CO}_{2}$ nos espaços vazios deixados pelo líquido iônico, mostra-se, na Figura 5.9, a função de distribuição espacial (SDF) de cátions e $\mathrm{CO}_{2}$ em torno do ânion (A) e ânion e $\mathrm{CO}_{2}$ em torno do cátion (B), obtidos de simulações MD a $313 \mathrm{~K}$.

5 Shiflett e Yokozeki (2010).

76 Prasad e Senapati (2009).

77 Haghbakhsh et al. (2013).

78 Hong et al. (2011).

79 Cadena et al. (2004). 

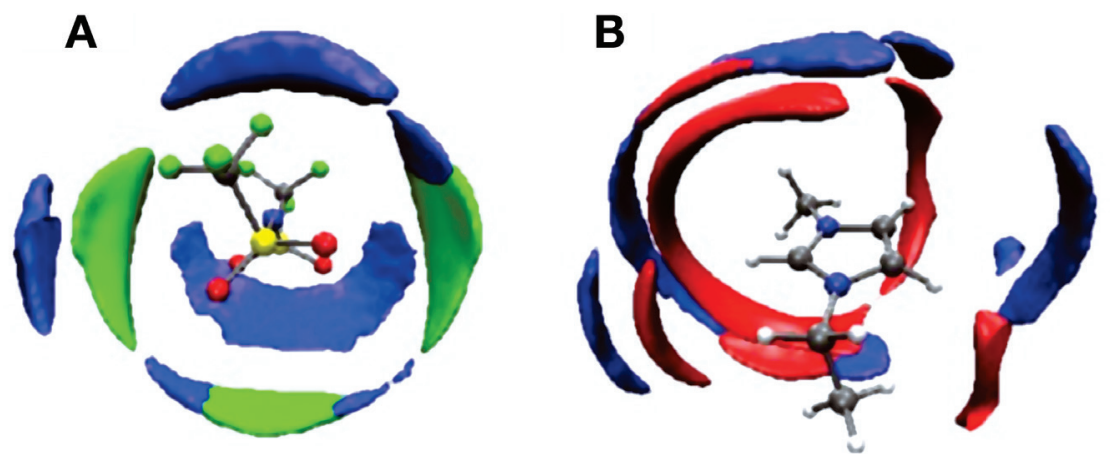

Figura 5.9 Função de distribuição espacial (SDF) de cátion (verde) e $\mathrm{CO}_{2}$ (azul) em torno do ânion (A) e de ânion (vermelho) e $\mathrm{CO}_{2}$ (azul) em torno do cátion (B).

A Figura 5.9 mostra a SDF para o sistema emim[Tf2N $] / \mathrm{CO}_{2}$, contendo 50 moléculas de $\mathrm{CO}_{2}$. Em recente trabalho também de simulação $\mathrm{MD}$, Yue et al. mostraram a SDF de $\mathrm{CO}_{2}$ em torno de cátion e ânion para os sistemas emim [Tf2 $\left.\mathrm{N}\right] / \mathrm{CO}_{2}$, emim $\left[\mathrm{PF}_{6}\right] / \mathrm{CO}_{2}$ e $\mathrm{PC}_{666,14}\left[\mathrm{PF}_{6}\right] / \mathrm{CO}_{2}$, porém não estabelecendo a correlação entre a baixa expansão do volume e o rearranjo dos líquidos iônicos para acomodarem o $\mathrm{CO}_{2}{ }^{80} \mathrm{~A}$ Figura 5.9A mostra que $\mathrm{CO}_{2}$ (azul) coordena os ânions em regiões em que não há cátions (verde), revelando, do ponto de vista microscópico, que o $\mathrm{CO}_{2}$ ocupa os vazios deixados pelos cátions. A Figura 5.9B mostra as ocorrências de ânions e $\mathrm{CO}_{2}$ em torno do cátion. Os ânions coordenam o cátion pelos sítios de interação de hidrogênio, mas isso evidencia que a interação mais favorável e de maior probabilidade ocorre com o H mais ácido do anel imidazólio. Nesse estudo, mostrou-se, conforme já destacado anteriormente por Lin e Freeman, ${ }^{81}$ que as cavidades existentes nos LIs são decorrências das interações mais fracas entre cátion-ânion e, portanto, reguladas pela termodinâmica dessas interações. Ou seja, quanto mais fraca a interação cátion-ânion, maior será a ocorrência de cavidades, permitindo, assim, a captura de gases como $\mathrm{CO}_{2}$. Esse mecanismo é de suma importância na modulação de líquidos iônicos capazes de atuar na captura e separação de gases.

\subsection{CONSIDERAÇõeS FINAIS}

Simulações de dinâmica molecular consistem em um método fundamental para compreender as observações experimentais e prever as propriedades de líquidos iônicos. Neste capítulo, apresentou-se uma visão geral sobre duas das mais importantes abordagens computacionais para a modelagem desses líquidos, em especial dos líquidos iônicos: a dinâmica molecular atomística e a dinâmica molecular

80 Yue et al. (2011).

81 Lin e Freeman (2005). 
coarse-grained. Por meio das aplicações apresentadas, mostrou-se o quanto tais técnicas são versáteis e eficientes. Nos modelos computacionais de líquidos iônicos, as estruturas, a dinâmica e a energética do líquido podem ser simuladas em resolução atomística e/ou em escalas espacial e temporal superiores. Esses modelos permitem compreender a origem e o comportamento molecular desses líquidos, além de prever suas propriedades macroscópicas.

\section{REFERÊNCIAS}

Allen, M. P.; Tildesley, D. J. Computer simulations of liquids. Oxford: Clarendon Press, 1987.

Anthony, J. L.; Anderson, J. L.; Maginn, E. J.; Brennecke, J. F. Anion effects on gas solubility in ionic liquids. J Phys Chem B, 109, p. 6366-6374, 2005.

ARIga, K.; Kunitake, T. Supramolecular chemistry. Fundamentals and applications: advanced textbook. 1. ed. Nova York: Springer, 2006.

Azevedo, A. M. O.; Ribeiro, D. M. G.; Pinto, P. C. A. G.; Lúcio, M.; Reis, S.; Saraiva, M. L. M. F. S. Imidazolium ionic liquids as solvents of pharmaceuticals: influence on hsa binding and partition coefficient of nimesulide. International Journal of Pharmaceutics, 443, 2013.

Barnoud, J.; Rossi, G.; Monticelli, L. Lipid membranes as solvents for carbon nanoparticles. Physical Review Letters, 112, 2014.

BARONE, V. (Ed.). Computational strategies for spectroscopy: from small molecules to nano systems. 1. ed. Londres: Wiley, 2011.

Bica, K.; Rodríguez, H.; Gurau, G.; Cojocaru, O. A.; Rissager, A.; Fehrmann, R.; Rogers, R. D. Pharmaceutically active ionic liquids with solids handling, enhanced thermal stability, and fast release. Chemical Communications, 48, p. 5422-5424, 2012.

Blanchard, L. A.; Hancu, D.; Beckman, E. J.; Brennecke, J. F. Green processing using ionic liquids and Co2. Nature, 399, p. 28-29, 1999.

Borodin, O.; SмIтH, G. D. Mechanism of ion transport in amorphous poly(ethylene oxide)/litfsi from molecular dynamics simulations. Macromolecules, 39, p. 1620-1629, $2006 \mathrm{a}$. . Development of many-body polarizable force fields for li-battery components: 1. ether, alkane, and carbonate-based solvents. J Phys Chem B, 110, p. 6279-6292, 2006b.

. Development of many-body polarizable force fields for li-battery applications: 2 . litfsi-doped oligoether, polyether, and carbonate-based electrolytes. J Phys Chem B, 110, p. 6293-6299, 2006c.

Brooks, A. Ionic liquids: synthesis, characterization and applications. Nova York: Nova Science Pub Inc., 2014. 
Cadena, C.; Anthony, J. L.; Shah, J. K.; Morrow, T. I.; Brennecke, J. F.; Maginn, E. J. Why is CO2 so soluble in imidazolium-based ionic liquids? J Am Chem Soc., 126, p. 53005308, 2004.

Canongia Lopes, J. N.; PÁdua, A. A. H. Molecular force field for ionic liquids iii: imidazolium, pyridinium, and phosphonium cations; chloride, bromide, and dicyanamide anions. Journal of Physical Chemistry B, 110, p. 19586-19592, 2006.

Case, D. A.; Darden, T. A.; Cheatham, T. E.; Simmerling, C. L.; Wang, Y.; Duke, R. E.; Luo, R.; Crowley, M.; Walker, R. C.; Zhang, W.; Merz, K. M.; Wang, B.; Hayik, S.; Roitberg, A.; Seabra, G.; Kolossváry, I.; Wong, K. F.; Paesani, F.; Vanicek, J.; Wu, X.; Brozell, S. R.; Steinbrecher, T.; Gohlke, H.; Yang, L.; Tan, C.; Mongan, J.; Hornak, V.; Cui, G.; Mathews, D. H.; Seetin, M. G.; Sagui, C.; Babin, V.; Kollman, P. A. Amber 10 user's manual. São Francisco: University of California, 2008.

Cojocaru, O. A.; Bica, K.; Gurau, G.; Narita, A.; McCrary, P. D.; Shamshina, J. L.; BArber, P. S.; Rogers, R. D. Prodrug ionic liquids: functionalizing neutral active pharmaceutical ingredients to take advantage of the ionic liquid form. MedChemComm, 4, p. 559-563, 2013.

Costa, L. T.; Ribeiro, M. C. Molecular dynamics simulation of polymer electrolytes based on poly(ethylene oxide) and ionic liquids. i. structural properties. J Chem Phys, 124, p. 184902-184908, 2006.

Duan, Y.; Halley, J. W.; Curtiss, L.; Redfern, P. Mechanisms of lithium transport in amorphous polyethylene oxide. J Chem Phys, 122, p. 54702-54708, 2005.

Ekkabut, J. W.; Baoukina, S.; Triampo, W.; Tang, I. M.; Tieleman, D. P.; Monticelli, L. Computer simulation study of fullerene translocation through lipid membranes. Nature Nanotechnology, 3, p. 363-368, 2008.

Elliott, S. R. Physics of amorphous materials. Nova York: Longman Scientific and Technical, 1952.

Feng, R.; ZhaO, D.; Guo, Y. Revisiting characteristics of ionic liquids: a review for further application development. J. Env. Protec., 1, 1, 95, p. 95-104, 2010.

Greaves, T. L.; Weerawardena, A.; Fong, C.; Krodkiewska, I.; Drummond, C. J. Protic ionic liquids: solvents with tunable phase behavior and physicochemical properties. J. Phys. Chem. B, 110, p. 22479-22487, 2006.

Haghbakhsh, R.; Soleymani, H.; Raeissi, S. A simple correlation to predict high pressure solubility of carbon dioxide in 27 commonly used ionic liquids. The Journal of Supercritical Fluids, 77, p. 158-166, 2013.

Hansen, J. P.; McDonald, I. R. Theory of simple liquids. Nova York: Academic Press, 1990. Hong, S. Y.; Im, J.; Palgunadi, J.; Lee, S. D.; Lee, J. S.; Kim, H. S.; Cheong, M.; Jung, K.-D. Ether-functionalized ionic liquids as highly efficient So2 absorbents. Energy \& Environmental Science, 4, p. 1802-1806, 2011. 
Hough, W. L.; Smiglak, M.; Rodríguez, H.; Swatloski, R. P.; Spear, S. K.; Daly, D. T.; Pernak, J.; Grisel, J. E.; Carliss, R. D.; Soutullo, M. D.; Davis, J. J. H.; Rogers, R. D. The third evolution of ionic liquids: active pharmaceutical ingredients. New Journal of Chemistry, 31, p. 1429-1436, 2007.

Huddleston, J. G.; Willauer, H. D.; Swatloski, R. P.; Visser, A. E.; Rogers, R. D. Room temperature ionic liquids as novel media for "clean" liquid-liquid extraction. Chemical Communications, 16, p. 1765-1766, 1998.

Huiyong, W.; Lamei, Z.; Jianji, W.; Zhiyong, L.; Suojiang, Z. The first evidence for unilamellar vesicle formation of ionic liquids in aqueous solutions. Chemical Communications, 49, 2013.

Hwang, H.; Schatz, G. C.; Ratner, M. A. Steered molecular dynamics studies of the potential of mean force of a na+ or $\mathrm{k}+$ ion in a cyclic peptide nanotube. J. Phys. Chem. B, 110, p. 26448-26460, 2006.

. Coarse-grained molecular dynamics study of cyclic peptide nanotube insertion into a lipid bilayer. J. Phys. Chem. A, 113, p. 4780-4787, 2009.

Hywel, D. W.; Yasemin, S.; Leigh, F.; Tri-Hung, N.; Peter, J. S.; Christopher, J. H. P. Ionic liquids provide unique opportunities for oral drug delivery: structure optimization and in vivo evidence of utility. Chemical Communications, 50, 2014.

Jorgensen, W. L.; Maxwell, D. S.; Tirado-Rives, J. Development and testing of the opls all-atom force field on conformational energetics and properties of organic liquids. Journal of the American Chemical Society, 118, p. 11225-11236, 1996.

LEACH, A. Molecular modelling: principles and applications. 2. ed. Nova York: Prentice Hall, 2001.

Lee, K.-Y.; Kim, C.-S.; Kim, H.-G.; Cheong, M.-S.; MukherJee, D. K.; Jung, K.-D. Effects of halide anions to absorb SO2 in ionic liquids. Bulletin of the Korean Chemical Society, 31, p. 1937-1940, 2010.

Li, J.-R.; Ma, Y.; McCarthy, M. C.; Sculley, J.; Yu, J.; Jeong, H.-K.; Balbuena, P. B.; Zhou, H.-C. Carbon dioxide capture-related gas adsorption and separation in metal-organic frameworks. Coordination Chemistry Reviews, 255, p. 1791-1823, 2011.

Lin, H.; Freeman, B. D. Materials selection guidelines for membranes that remove CO2 from gas mixtures. Journal of Molecular Structure, 739, p. 57-74, 2005.

Lopes, J.; PÁduA, A. A. H. Nanostructural organization in ionic liquids. Journal of Physical Chemistry B, 110, p. 3330-3335, 2006.

Lourenço, T. C.; Coelho, M. F.; Ramalho, T. C.; Van der Spoel, D.; Costa, L. T. Insights on the solubility of CO2 in 1-Ethyl-3-Methylimidazolium bis(trifluoromethylsulfonyl) imide from the microscopic point of view. Environ Sci Technol, 47, p. 7421-7429, 2013.

Lynden-Bell, R. M.; Del Popolo, M. G.; Youngs, T. G. A.; Kohanoff, J.; Hanke, C. G.; Harper, J. B.; Pinilla, C. C. Simulations of ionic liquids, solutions, and surfaces. Accounts of Chemical Research, 40, p. 1138-1145, 2007. 
MacKerell, A. D.; Bashford, D.; Bellott, M.; Dunbrack, R. L.; Evanseck, J. D.; Field, M. J.; Fischer, S.; GaO, J.; Guo, H.; Ha, S.; Joseph-McCarthy, D.; Kuchnir, L.; Kuczera, K.; Lau, F. T. K.; Mattos, C.; Michnick, S.; Ngo, T.; Nguyen, D. T.; Prodhom, B.; Reiher, W. E.; Roux, B.; Schlenkrich, M.; Smith, J. C.; Stote, R.; Straub, J.; WataNABE, M.; Wiórkiewicz-Kuczera, J.; Yin, D.; KARPlus, M. All-atom empirical potential for molecular modeling and dynamics studies of proteins. J. Phys. Chem. B, 102, 1998.

March, N. H.; Tosi, M. P. Atomic dynamics in liquids. Nova York: Dover Publications, 1976. Marrink, S. J.; Risselada, H. J.; Yefimov, S.; Tieleman, D. P.; Vries, A. H. The MARTINI force field: coarse grained model for biomolecular simulations. J. Phys. Chem. B, 111, p. 7812-7824, 2007.

Martins, S.; Fedorov, A.; Afonso, C. A. M.; Baleizao, C.; Berberan-Santos, M. N. Fluorescence of fullerene $\mathrm{C}_{70}$ in ionic liquids. Chemical Physics Letters, 497, p. 43-47, 2010. McCrary, P. D.; Beasley, P. A.; Gurau, G.; Narita, A.; Barber, P. S.; Cojocaru, O. A.; Rogers, R. D. Drug specific, tuning of an ionic liquid's hydrophilic-lipophilic balance to improve water solubility of poorly soluble active pharmaceutical ingredients. New Journal of Chemistry, 37, p. 2196-2202, 2013.

Mchedlov-Petrossyan, N. O. Fullerenes in molecular liquids. Solutions in "good" solvents: another view. Journal of Molecular Liquids, 161, p. 1-12, 2011.

McQuarrie, D. A. Statistical mechanics. Sausalito: University Science Book, 2000.

Merlet, C.; Salanne, M.; Rotenberg, B.; Madden, P. A. Imidazolium ionic liquid interfaces with vapor and graphite: interfacial tension and capacitance from coarse-grained molecular simulations. Journal of Physical Chemistry C, 115, p. 16613-16618, 2011.

Mohammad, A.; Inamuddin, D. Green solvents I: properties and applications in Chemistry. Nova York: Springer, 2014.

Pádua, A. G. H.; Gomes, M. F. C.; Canongia Lopes, J. N. A. Molecular solutes in ionic liquids: a structural perspective. Accounts of Chemical Research, 40, p. 1087-1096, 2007.

Prasad, B. R.; Senapati, S. Explaining the differential solubility of flue gas components in ionic liquids from first-principle calculations. J Phys Chem B, 113, p. 4739-4743, 2009.

Rao, K. S.; Soonyong, S.; Arvind, K. Vesicles and reverse vesicles of an ionic liquid in ionic liquids. Chemical Communications, 49, 2013.

RAPAPORT, R. C. The art of molecular dynamics simulation. Cambridge: Cambridge University Press, 1995.

Reichardt, C. Polarity of ionic liquids determined empirically by means of solvatochromic pyridinium N-phenolate betaine dyes. Green Chemistry, 7, p. 339-351, 2005.

SAdus, R. J. Molecular simulations of fluids: theory, algorithms, and object-orientation. Nova York: Elsevier, 1999.

Sansom, M. S. P.; Biggin, P. C.; Neidle, S. Molecular simulations and biomembranes: from biophysics to function. Cambridge: Royal Society of Chemistry, 2010.

Schmid, G. Nanoparticles: from theory to application. 2. ed. Nova York: Wiley-VCH, 2010. 
Scott, V.; Gilfillan, S.; Markusson, N.; Chalmers, H.; Haszeldine, R. S. Last chance for carbon capture and storage. Nature Climate Change, 3, p. 105-111, 2012.

Scrivens, W. A.; Tour, J. M. Potent solvents for $\mathrm{C}_{60}$ and their utility for the rapid acquisition of C-13 NMR data for fullerenes. J. Chem. Soc. Chem. Comm., p. 1207-1109, 1993.

Semenov, K. N.; Charykov, N. A. Temperature dependence of solubility of individual light fullerenes and industrial fullerene mixture in 1-chloronaphthalene and 1-bromonaphthalene. J. Chem. Eng. Data, 55, p. 2373-2378, 2010.

Semenov, K. N.; Charykov, N. A.; Keskinov, V. A.; Piartman, A. K.; Blokhin, A. A.; Kopyrin, A. A. Solubility of light fullerenes in organic solvents. J. Chem. Eng. Data, 55, p. 13-36, 2010.

Senn, H. M.; Thiel, W. QM/MM methods for biomolecular systems. Angew. Chem. Int. Ed. Engl., 48, p. 1198-1229, 2009.

Shiflett, M. B.; Yokozeki, A. Solubilities and diffusivities of carbon dioxide in ionic liquids: [bmim][Pf6] and [bmim][Bf4]. Industrial \& Engineering Chemistry Research, 44, p. 4453-4464, 2005.

. Separation of $\mathrm{CO} 2$ and $\mathrm{H} 2 \mathrm{~S}$ using room-temperature ionic liquid [bmim][Pf6]. Fluid Phase Equilibria, 294, p. 105-113, 2010.

SHIM, Y.; KIM, H. J. Solvation of carbon nanotubes in a room-temperature ionic liquid. Acs Nano, 3, p. 1693-1702, 2009.

Siqueira, L. J.; Ribeiro, M. C. Molecular dynamics simulation of the polymer electrolyte poly(ethylene oxide)/liclo(4). ii. dynamical properties. J Chem Phys, 125, p. 214903214908, 2006.

- Molecular dynamics simulation of the ionic liquid n-ethyl-n,n-dimethyl-n-(2-methoxyethyl)ammonium bis(trifluoromethanesulfonyl)imide. J Phys Chem B, 111, p. 11776-11785, 2007.

Soares, T. A.; Hunenberger, P. H.; Kastenholz, M. A.; Krautler, V.; Lenz, T.; Lins, R. D.; Oostenbrink, C.; Van Gunsteren, W. F. An improved nucleic acid parameter set for the gromos force field. J Comput Chem, 26, p. 725-737, 2005.

Srivastava, A.; Voth, G. A. Hybrid approach for highly coarse-grained lipid bilayer models. J. Chem. Theory Comput., 9, p. 750-765, 2013.

Stoimenovski, J.; Dean, P. M.; Izgorodina, E. I.; MacFarlane, D. R. Protic pharmaceutical ionic liquids and solids: aspects of protonics. Faraday Discussions, 154, p. 439-464, 2012.

Suruchi, M.; Rabia, S.; Rakesh Kumar, M. An investigation of drug binding ability of a surface active ionic liquid: micellization, electrochemical, and spectroscopic studies. Langmuir, 28, 2012.

Talukdar, S.; Pradhan, P.; BanerJi, A. Electron donor-acceptor interactions of $\mathrm{C}_{60}$ with $\mathrm{N}$ - and P-donors: a rational approach towards its solubility. Fullerene Sci. Techn., 5, p. 547557, 1997. 
Urahata, S. M.; Ribeiro, M. C. Structure of ionic liquids of 1-alkyl-3-methylimidazolium cations: a systematic computer simulation study. J Chem Phys, 120, p. 1855-1863, 2004.

. Single particle dynamics in ionic liquids of 1-alkyl-3-methylimidazolium cations. J Chem Phys, 122, p. 024511-024519, 2005.

. Collective excitations in an ionic liquid. J Chem Phys, 124, p. 74513-74518, 2006.

Vishal Govind, R.; Chiranjib, B.; Surajit, G.; Sarthak, M.; Jagannath, K.; NilmoNI, S. A step toward the development of high-temperature stable ionic liquid-in-oil microemulsions containing double-chain anionic surface active ionic liquid. The Journal of Physical Chemistry B, 117, 2013.

Voth, G. A. E. Coarse-graining of condensed phase and biomolecular systems. [S.1.]: CRC Press, 2012.

WANG, Y.; JiAng, W.; YAn, T.; Voth, G. A. Understanding ionic liquids through atomistic and coarse-grained molecular dynamics simulations. Acc. Chem. Res., 40, p. 1193-1199, 2007.

WANG, Y.; Voth, G. A. Unique spatial heterogeneity in ionic liquids. Journal of the American Chemical Society, 127, p. 12192-12193, 2005.

WeINGäRTnER, H. Understanding ionic liquids at the molecular level: facts, problems, and controversies. Angew. Chem. Int. Ed., 47, p. 654-670, 2008.

Welton, T. Room-temperature ionic liquids: solvents for synthesis and catalysis. Chemical Reviews, 99, p. 2071-2083, 1999.

Yue, Z.-G.; Liu, X.-M.; ZhaO, Y.-L.; Zhang, X.-C.; Lu, X.-M.; Zhang, S.-J. Molecular simulation on microstructure of ionic liquids in capture of CO2. Chin. J. Proc. Eng., 11, p. $652-659,2011$.

ZHANG, X.; LIU, Z.; WANG, W. Screening of ionic liquids to capture CO2 by Cosmo-Rs and experiments. AIChE Journal, 54, p. 2717-2728, 2008. 